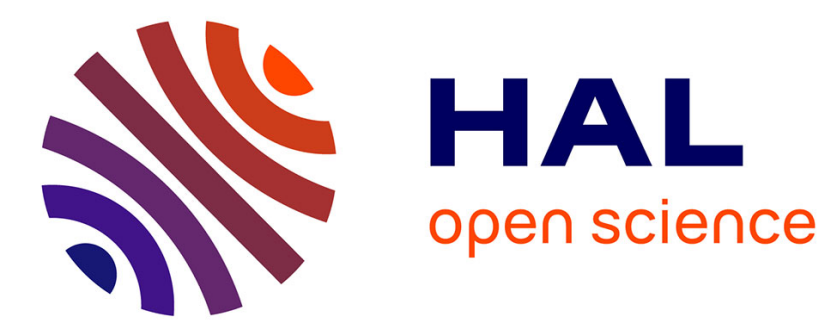

\title{
NEGATIVE RESULTS IN COCONVEX APPROXIMATION OF PERIODIC FUNCTIONS
}

German Dzyubenko, Victoria Voloshina, Lyudmyla Yushchenko

\section{To cite this version:}

German Dzyubenko, Victoria Voloshina, Lyudmyla Yushchenko. NEGATIVE RESULTS IN COCONVEX APPROXIMATION OF PERIODIC FUNCTIONS. Journal of Approximation Theory, 2021, 10.1016/j.jat.2021.105582 . hal-02956219

\section{HAL Id: hal-02956219 https://hal.science/hal-02956219}

Submitted on 2 Oct 2020

HAL is a multi-disciplinary open access archive for the deposit and dissemination of scientific research documents, whether they are published or not. The documents may come from teaching and research institutions in France or abroad, or from public or private research centers.
L'archive ouverte pluridisciplinaire HAL, est destinée au dépôt et à la diffusion de documents scientifiques de niveau recherche, publiés ou non, émanant des établissements d'enseignement et de recherche français ou étrangers, des laboratoires publics ou privés. 


\title{
NEGATIVE RESULTS IN COCONVEX APPROXIMATION OF PERIODIC FUNCTIONS
}

\author{
GERMAN DZYUBENKO, VICTORIA VOLOSHINA, \\ AND LYUDMYLA YUSHCHENKO
}

Abstract. We prove, that for each $r \in \mathbb{N}, n \in \mathbb{N}$ and $s \in \mathbb{N}$ there are a collection $\left\{y_{i}\right\}_{i=1}^{2 s}$ of points $y_{2 s}<y_{2 s-1}<\cdots<y_{1}<y_{2 s}+2 \pi=$ : $y_{0}$ and a $2 \pi$ - periodic function $f \in C^{(\infty)}(\mathbb{R})$, such that

$$
f^{\prime \prime}(t) \prod_{i=1}^{2 s}\left(t-y_{i}\right) \geq 0, \quad t \in\left[y_{2 s}, y_{0}\right]
$$

and for each trigonometric polynomial $T_{n}$ of degree $\leq n$ (of order $\leq$ $2 n+1)$, satisfying

$$
T_{n}^{\prime \prime}(t) \prod_{i=1}^{2 s}\left(t-y_{i}\right) \geq 0, \quad t \in\left[y_{2 s}, y_{0}\right]
$$

the inequality

$$
n^{r-1}\left\|f-T_{n}\right\|_{C(\mathbb{R})} \geq c_{r}\left\|f^{(r)}\right\|_{C(\mathbb{R})}
$$

holds, where $c_{r}>0$ is a constant, depending only on $r$. Moreover, we prove, that for each $r=0,1,2$ and any such collection $\left\{y_{i}\right\}_{i=1}^{2 s}$ there is a $2 \pi$ - periodic function $f \in C^{(r)}(\mathbb{R})$, such that $(-1)^{i-1} f$ is convex on $\left[y_{i}, y_{i-1}\right], 1 \leq i \leq 2 s$, and, for each sequence $\left\{T_{n}\right\}_{n=0}^{\infty}$ of trigonometric polynomials $T_{n}$, satisfying (2), we have

$$
\limsup _{n \rightarrow \infty} \frac{n^{r}\left\|f-T_{n}\right\|_{C(\mathbb{R})}}{\omega_{4}\left(f^{(r)}, 1 / n\right)}=+\infty,
$$

where $\omega_{4}$ is the fourth modulus of continuity.

\section{INTRODUCTION AND THE MAIN RESULTS}

Let $s \in \mathbb{N}$ and $\mathbb{Y}_{s}:=\left\{Y_{s}\right\}$, where the collections $Y_{s}=\left\{y_{i}\right\}_{i=1}^{2 s}$ of points $y_{i} \in \mathbb{R}$ are such that $y_{2 s}<\cdots<y_{1}<y_{2 s}+2 \pi=$ : $y_{0}$. We say that a $2 \pi$-periodic function $f \in C(\mathbb{R})$ is piecewise convex with respect to $Y_{s}$, if it is a convex function on $\left[y_{1}, y_{0}\right]$ and changes its convexity at the points $Y_{s}$, that is, if $(-1)^{i-1} f$ is convex on $\left[y_{i}, y_{i-1}\right], 1 \leq i \leq 2 s$. We denote by $\Delta^{(2)}\left(Y_{s}\right)$ the collection of all such piecewise convex functions. Note that if, in addition, $f \in C^{(2)}(\mathbb{R})$, then $f \in \Delta^{(2)}\left(Y_{s}\right)$, if and only if,

$$
f^{(2)}(t) \prod_{i=1}^{2 s}\left(t-y_{i}\right) \geq 0, \quad t \in\left[y_{2 s}, y_{0}\right] \text {. }
$$

2010 Mathematics Subject Classification. 42A05, 42A10, 41A17, 41A25, 41A29.

Key words and phrases. shape preserving approximation, trigonometric polynomial, Jackson, convex. 
Denote by $C^{(r)}, r \in \mathbb{N}$, the space of $2 \pi$ - periodic functions $f \in C^{(r)}(\mathbb{R})$. We also need the notation $W^{r}, \quad r \in \mathbb{N}$, for the Sobolev space of $2 \pi$-periodic functions $f \in A C^{(r-1)}(\mathbb{R})$, such that

$$
\left\|f^{(r)}\right\|<+\infty
$$

where

$$
\|g\|:=\operatorname{esssup}_{x \in \mathbb{R}}|g(x)| .
$$

If, in addition, $g$ is continuous, then, of course,

$$
\|g\|=\sup _{x \in \mathbb{R}}|g(x)| .
$$

Let $\mathbb{T}_{n}$ be the space of trigonometric polynomials of degree $\leq n$ (of order $2 n+1)$ and, for the function $g \in \Delta^{(2)}\left(Y_{s}\right)$, denote by

$$
E_{n}^{(2)}\left(g, Y_{s}\right):=\inf _{T_{n} \in \mathbb{T}_{n} \cap \Delta^{(2)}\left(Y_{s}\right)}\left\|g-T_{n}\right\|,
$$

the error of the best coconvex approximation of the function $g$. It is known [10] that if $f \in \Delta^{(2)}$, then

$$
E_{n}^{(2)}\left(f, Y_{s}\right) \leq c(s) \omega_{3}(f, 1 / n), \quad n \geq N
$$

where $c(s)$ is a constant, depending only on $s, N$ is a number, depending only on $Y_{s}$, and

$$
\omega_{k}(f, t)=\sup _{h \in[0, t]}\left\|\sum_{j=0}^{k}(-1)^{k-j}\left(\begin{array}{l}
k \\
j
\end{array}\right) f(\cdot+i h)\right\|, \quad t \geq 0,
$$

is the modulus of continuity of a function $f$ of order $k \in \mathbb{N}$. For each $f \in \Delta^{(2)}\left(Y_{s}\right)(1.1)$ implies

$$
\begin{aligned}
& E_{n}^{(2)}\left(f, Y_{s}\right) \leq \frac{c(s)}{n} \omega_{2}\left(f^{\prime}, 1 / n\right), \quad n \geq N, \quad \text { if } \quad f \in C^{(1)}, \\
& E_{n}^{(2)}\left(f, Y_{s}\right) \leq \frac{c(s)}{n^{2}} \omega_{1}\left(f^{\prime \prime}, 1 / n\right), \quad n \geq N, \quad \text { if } \quad f \in C^{(2)},
\end{aligned}
$$

and

$$
E_{n}^{(2)}\left(f, Y_{s}\right) \leq \frac{c(s, r)}{n^{r}}\left\|f^{(r)}\right\|, \quad n \geq N, \quad \text { if } \quad f \in W^{r},
$$

$1 \leq r \leq 3$, where $N$ is a number, depending only on $Y_{s}$. Leviatan, Motorna and Shevchuk [5] conjectured, that (1.4) holds for all $r \in \mathbb{N}$.

However it turns out, that all these estimates in general are invalid with $N$ independent of $Y_{s}$. In other words, unlike the classical Jackson inequality, (1.4) cannot be had with $N=1$. So, our first result is 
Theorem 1.1. Let $r \in \mathbb{N}$ and $s \in \mathbb{N}$ be given. For each $n \geq 1$ there are $a$ collection $Y_{s} \in \mathbb{Y}_{s}$ and a function $f \in \Delta^{(2)}\left(Y_{s}\right) \cap W^{r}$, such that

$$
E_{n}^{(2)}\left(f, Y_{s}\right)>\frac{c(r)}{n^{r-1}}\left\|f^{(r)}\right\|
$$

where $c(r)=$ const $>0$ depends only on $r$.

Moreover, (1.3) cannot be improved by replacing $\omega_{1}$ with $\omega_{k}, k \geq 4$. Our second main result is

Theorem 1.2. For each $Y_{s} \in \mathbb{Y}$ there is a function $f \in \Delta^{(2)}\left(Y_{s}\right) \cap C^{(2)}$, such that

$$
\limsup _{n \rightarrow \infty} \frac{n^{2} E_{n}^{(2)}\left(f, Y_{s}\right)}{\omega_{4}\left(f^{\prime \prime}, 1 / n\right)}=+\infty
$$

Clearly, $f \neq$ const in all Theorems in this paper. For the completeness we formulate an easy corollary of Example 1 in [2].

Theorem 1.3. For each $Y_{s} \in \mathbb{Y}$ there is a function $f \in \Delta^{(2)}\left(Y_{s}\right) \cap C^{(1)}$, such that

$$
\limsup _{n \rightarrow \infty} \frac{n E_{n}^{(2)}\left(f, Y_{s}\right)}{\omega_{3}\left(f^{\prime}, 1 / n\right)}=+\infty
$$

whence

$$
\limsup _{n \rightarrow \infty} \frac{E_{n}^{(2)}\left(f, Y_{s}\right)}{\omega_{4}(f, 1 / n)}=+\infty .
$$

We believe that Theorems $1.1-1.3$ cover all negative results in the question of the validity of Jackson type estimates in the coconvex approximation of periodic functions. In particular, we conjecture, that for each $k \in \mathbb{N}, r \in \mathbb{N}, r \geq 3, s \in \mathbb{N}$ and $f \in \Delta^{(2)}\left(Y_{s}\right) \cap C^{(r)}$ we have

$$
E_{n}^{(2)}\left(f, Y_{s}\right) \leq \frac{c(k, r, s)}{n^{r}} \omega_{k}\left(f^{(r)}, 1 / n\right), \quad n \geq N\left(k, r, Y_{s}\right) .
$$

In other words, we conjecture, that the truth table of the validity of Jackson type estimates in the coconvex approximation of periodic functions has the same form as the truth table of Jackson type estimates in the coconvex approximation of non-periodic functions by algebraic polynomials, see [6], Page 114, Fig. 3, or [4], Page 62, Table 24.

Remark 1.4. We do not discuss the comonotone approximation in the Introduction, we only note, that in the comonotone approximation of periodic functions more positive, but less negative results are known, see, [7], [8], [1] for more details. However in the last Section we formulate the analogs of Theorems 1.2 and 1.3 for the comonotone (co-1-monotone) approximation. We do not discuss also co- $q$-monotone approximation of periodic functions for $q>2$, since, in opposite to coconvex (co-2-monotone) approximation, the Jackson type estimates are invalid for all parameters, even if we allow both constants $c$ and $N$ in (1.8) to depend on $f$. This is recently proved by Leviatan, Motorna and Shevchuk [5]. 
We prove Theorem 1.1 in the next Section, and Theorems 1.2 and 1.3 in the last Section. In the proofs we apply the ideas from [3] and we have to overcome the constrains and challenges of periodicity.

\section{Proof of Theorem 1.1}

We begin with the following Lemma 2.1

Lemma 2.1. Let an integer $n \in \mathbb{N}$ and a positive number $\delta \leq \frac{1}{n}$ be given. If a polynomial $T \in \mathbb{T}_{n}$ satisfies

$$
T^{\prime}( \pm \delta)=0 \quad \text { and } \quad T^{\prime}(t) \geq 0, \quad \text { for } \quad \delta \leq|t| \leq \pi
$$

then

$$
T^{\prime}(t) \equiv 0 .
$$

Proof. Assume to the contrary, that (2.1) is invalid. Then without loss of generality we assume that $\left\|T^{\prime}\right\|=1$. Put $\tau:=T^{\prime}$. By Bernstein inequality, $\left\|\tau^{\prime}\right\| \leq n$ and $\left\|\tau^{\prime \prime}\right\| \leq n^{2}$. Let $t_{0} \in[-\delta, 2 \pi-\delta]$ be a point, such that $\left|\tau\left(t_{0}\right)\right|=\|\tau\|=1$. Since, for $t \in[-\delta, \delta]$,

$$
\begin{aligned}
|\tau(t)| & =\left|\tau(t)+\frac{t-\delta}{2 \delta} \tau(-\delta)-\frac{t+\delta}{2 \delta} \tau(\delta)\right|=\frac{1}{2}\left|\tau^{\prime \prime}(\theta)\right|(\delta-t)(\delta+t) \\
& \leq \frac{1}{2} n^{2}\left(\delta^{2}-t^{2}\right)<1,
\end{aligned}
$$

where $\theta \in(-\delta, \delta)$, we conclude, that $t_{0} \notin[-\delta, \delta]$, whence $\tau\left(t_{0}\right)=1$. Then, $1-\tau(t)=\tau\left(t_{0}\right)-\tau(t) \leq n\left|t-t_{0}\right|, \quad$ that is $\quad \tau(t) \geq 1-n\left|t-t_{0}\right|, \quad t \in \mathbb{R}$, that implies

and

$$
(-\delta, \delta) \cap\left(t_{0}-1 / n, t_{0}+1 / n\right)=\emptyset
$$

Therefore

$$
\int_{t_{0}-1 / n}^{t_{0}+1 / n} \tau(t) d t \geq \frac{1}{n}
$$

$$
\begin{aligned}
0 & =T(2 \pi-\delta)-T(-\delta)=\int_{-\delta}^{2 \pi-\delta} \tau(t) d t \geq \int_{t_{0}-1 / n}^{t_{0}+1 / n} \tau(t) d t+\int_{-\delta}^{\delta} \tau(t) d t \\
& \geq \frac{1}{n}-\frac{1}{2} n^{2} \int_{-\delta}^{\delta}\left(\delta^{2}-t^{2}\right) d t=\frac{1}{n}-\frac{2}{3} n^{2} \delta^{3} \geq \frac{1}{3 n} \neq 0
\end{aligned}
$$

- a contradiction.

Proof of Theorem 1.1. Let $\tilde{S} \in C^{(\infty)}(\mathbb{R})$, be a monotone odd function, such that $\tilde{S}(x)=\operatorname{sgn} x,|x| \geq 1$, and

$$
S(x):=\frac{1}{2}+\frac{1}{2} \tilde{S}(x) .
$$

Put

$$
s_{j}:=\left\|S^{(j)}\right\|, \quad j \in \mathbb{N}_{0}
$$


Take

$$
\begin{gathered}
h:=\frac{1}{3 n}, \\
y_{2 s}=-3 h, \quad y_{1}=3 h,
\end{gathered}
$$

and, if $s \geq 2$, then let $-h \leq y_{2 s-1}<. .<y_{2} \leq h$, say

$$
y_{i}=h-2 h \frac{i-2}{2 s-3}, \quad i=2, \ldots, 2 s-1 .
$$

We will prove, that the desired $2 \pi$ periodic function $f$ can be taken in the form

$$
f(x):=\int_{0}^{x} f^{\prime}(t) d t
$$

where $f^{\prime}$ is a $2 \pi$-periodic odd function, defined on $[0, \pi]$ by

$$
f^{\prime}(t):= \begin{cases}-S\left(\frac{t-2 h}{h}\right), & \text { if } t \in[0,1], \\ S\left(\frac{t-\pi+h}{h}\right)-1, & \text { if } t \in[1, \pi] .\end{cases}
$$

Note that $f^{\prime}(t)=-1$ for $3 h \leq t \leq \pi-2 h$. Clearly, $f \in \Delta^{(2)}\left(Y_{s}\right) \cap W^{r}$. Since $3 h=\frac{1}{n}$, Lemma 2.1 yields $T_{n}^{\prime \prime} \equiv 0$, if $T_{n} \in \Delta^{(2)}\left(Y_{s}\right) \cap \mathbb{T}_{n}$, hence (the periodic function) $T_{n} \equiv$ const. Therefore

$$
E_{n}^{(2)}\left(f, Y_{s}\right) \geq \frac{1}{2}(f(0)-f(\pi))=-\frac{1}{2} f(\pi) \geq \frac{1}{2}(\pi-5 h)>\frac{2}{3} .
$$

Thus,

$$
\frac{E_{n}^{(2)}\left(f, Y_{s}\right)}{\left\|f^{(r)}\right\|}=E_{n}^{(2)}\left(f, Y_{s}\right) \frac{h^{r-1}}{s_{r-1}} \geq \frac{2 h^{r-1}}{3 s_{r-1}}=\frac{2}{3^{r} s_{r-1} n^{r-1}} .
$$

Theorem 1.1 is proved with $c(r) \geq \frac{2}{3^{r} s_{r-1}}$.

\section{Auxiliary Results}

Denote by $\hat{S}$ an even function $\hat{S} \in C^{(\infty)}(\mathbb{R})$, such that $x \hat{S}^{\prime}(x) \geq 0, x \in \mathbb{R}$, and

$$
\hat{S}(x)=\left\{\begin{array}{lll}
0, & \text { if } \quad|x| \leq 1 \\
1, & \text { if } \quad|x| \geq 2 .
\end{array}\right.
$$

Put $\hat{s}_{j}:=\left\|\hat{S}^{(j)}\right\|, \quad j \in \mathbb{N}$. Fix a positive number $d \leq \pi / 4$ and for each positive $b \leq d / 2$ denote by $q_{b}$ and $g_{b}$ the $2 \pi$-periodic functions, such that

$$
q_{b}(x):=(1-\hat{S}(x / d))(\cos b-\cos x) \sin x, \quad x \in[-\pi, \pi],
$$

and

$$
g_{b}(x):=\hat{S}(x / b) q_{b}(x), \quad x \in[-\pi, \pi] .
$$

Clearly, $q_{b}$ and $g_{b}$ are odd functions,

$$
\left\|g_{b}\right\|<1, \quad\left\|q_{b}\right\|<1,
$$

and for each collection $Y=\left\{y_{i}\right\}_{i=1}^{2 s}$, such that

$$
y_{2}+2 d \leq y_{1}=0 \leq y_{0}-2 d,
$$


we have

$$
g_{b}(t) \prod_{i=1}^{2 s}\left(t-y_{i}\right) \geq 0, \quad t \in\left[y_{2 s}, y_{0}\right] .
$$

Now, for a function $f \in C[a, b]$ we denote by

$$
\|f\|_{[a, b]}:=\|f\|_{C[a, b]}=\max _{x \in[a, b]}|f(x)|
$$

and formulate Lemma 3.1, which is a particular case of Privalov Theorem (see, e.g., [9], pg. 96-98.)

Lemma 3.1. For each polynomial $T_{n} \in \mathbb{T}_{n}$ and any positive number $h \leq \pi$, we have

$$
h\left|T_{n}^{\prime}(0)\right| \leq c n\left\|T_{n}\right\|_{[-h, h]} .
$$

Here and in the sequel $c$ stand for different absolute positive constants. We conclude the Section with the following

Lemma 3.2. We have

$$
\omega_{4}\left(g_{b}, t\right) \leq c_{1}\left(b^{3}+(t / d)^{4}\right),
$$

and for each polynomial $T_{n} \in \mathbb{T}_{n}$, satisfying $T_{n}^{\prime}(0) \geq 0$, and a positive number $h \leq d$,

$$
\left\|g_{b}-T_{n}\right\|_{[-h, h]} \geq c_{2} \frac{h b^{2}}{n}-3 b^{3} .
$$

where $c_{1}$ and $c_{2} \leq 1$ are positive absolute constants.

Proof. First we show, that

$$
\left\|g_{b}-q_{b}\right\| \leq 3 b^{3}
$$

Indeed, if $|x| \leq 2 b$, then

$$
\begin{aligned}
\left|g_{b}(x)-q_{b}(x)\right| & =(1-\hat{S}(x / b))|(\cos b-\cos x) \sin x| \\
& \leq 2\left|\sin \frac{x-b}{2} \sin \frac{x+b}{2} \sin x\right| \leq \frac{1}{2}\left|x\left(x^{2}-b^{2}\right)\right| \leq 3 b^{3} .
\end{aligned}
$$

If otherwise $2 b \leq|x| \leq \pi$, then $g_{b}(x)=q_{b}(x)$, so (3.7) holds.

To prove $(3.5)$ we note, that the equality $\left\|\hat{S}^{(j)}(\cdot / d)\right\|=\hat{s}_{j} d^{-j}$ yields $\left\|q_{b}^{(4)}\right\| \leq c d^{-4}$. Therefore

$\omega_{4}\left(g_{b}, t\right) \leq \omega_{4}\left(g_{b}-q_{b}, t\right)+\omega_{4}\left(q_{b}, t\right) \leq 2^{4}\left\|g_{b}-q_{b}\right\|+t^{4}\left\|q_{b}^{(4)}\right\| \leq 48 b^{3}+c d^{-4} t^{4}$, that implies (3.5).

Finally, to prove (3.6), we apply Lemma 3.1. Since $q_{b}(x)=(\cos b-$ $\cos x) \sin x$ for $x \in[-d, d]$, we get

$$
\begin{aligned}
2 \sin ^{2} \frac{b}{2} & =-q_{b}^{\prime}(0) \leq T_{n}^{\prime}(0)-q_{b}^{\prime}(0) \leq \frac{c n}{h}\left\|T_{n}-q_{b}\right\|_{[-h, h]} \\
& \leq \frac{c n}{h}\left(\left\|T_{n}-g_{b}\right\|_{[-h, h]}+\left\|q_{b}-g_{b}\right\|\right) \leq \frac{c n}{h}\left(\left\|T-g_{b}\right\|_{[-h, h]}+3 b^{3}\right),
\end{aligned}
$$


that yields (3.6).

\section{NeGATIVE RESUlt in COPOSITIVE APPROXIMATION}

For each collection $Y_{s}=\left\{y_{i}\right\}_{i=1}^{2 s} \in \mathbb{Y}_{s}$ denote by $\Delta^{(0)}\left(Y_{s}\right)$ the set of all $2 \pi$-periodic functions $f \in C(\mathbb{R})$, such that

$$
f(t) \prod_{i=1}^{2 s}\left(t-y_{i}\right) \geq 0, \quad t \in\left[y_{2 s}, y_{0}\right] .
$$

For the function $g \in \Delta^{(0)}\left(Y_{s}\right)$, denote by

$$
E_{n}^{(0)}\left(g, Y_{s}\right):=\inf _{T_{n} \in \mathbb{T}_{n} \cap \Delta(0)\left(Y_{s}\right)}\left\|g-T_{n}\right\|,
$$

the error of the best copositive approximation of the function $g$. We prove

Theorem 4.1. For each $Y_{s} \in \mathbb{Y}_{s}$ there is a function $f \in \Delta^{(0)}\left(Y_{s}\right)$, such that

$$
\limsup _{n \rightarrow \infty} \frac{E_{n}^{(0)}\left(f, Y_{s}\right)}{\omega_{4}(f, 1 / n)}=+\infty
$$

and

$$
\int_{-\pi}^{\pi} f(x) d x=0
$$

Proof. Without loss of generality assume, that $y_{1}=0$. Put $d:=\frac{1}{2} \min \left\{y_{0},-y_{2}, \pi / 2\right\}$, so that (3.3) implies, for all positive $b \leq d / 2$,

$$
g_{b} \in \Delta^{(0)}\left(Y_{s}\right),
$$

where $g_{b}$ is defined in the previous Section. Following [3], pg.343-345, we put

$$
b_{n}:=\left(\frac{1}{n}\right)^{\frac{4}{3}}, \quad f_{n}(x)=g_{b_{n}}(x),
$$

and note that (3.5) implies, for all $n \geq(2 / d)^{3 / 4}$, so that $2 b_{n} \leq d$,

$$
\omega_{4}\left(g_{b} ; t\right) \leq c_{1}\left(1+d^{-4}\right) t^{4}, \quad t \geq \frac{1}{n} .
$$

We are now in a position to define the desired in Theorem 4.1 function $f$.

First we put $\varepsilon=0.1$ and choose $n_{0} \geq(2 / d)^{3 / 4}$, so big that

$$
c_{1}\left(1+d^{-4}\right)<n_{0}^{\varepsilon} \text {. }
$$

Set $d_{0}:=d$ and

$$
d_{j}:=\frac{c_{2}}{4} \frac{b_{n_{j-1}} b_{n_{j}}^{2}}{n_{j}} d_{j-1}, \quad j \geq 1,
$$


where the increasing sequence $\left\{n_{\nu}\right\}$ is defined by induction as follows. Suppose that $\left\{n_{0}, \ldots, n_{\sigma-1}\right\}$ have been defined, then put

$$
F_{\sigma-1}(x):=\sum_{j=1}^{\sigma-1} d_{j-1} f_{n_{j}}(x), \quad\left(F_{0}(x): \equiv 0\right),
$$

and take $n_{\sigma}>n_{\sigma-1}$ so big that

$$
\left\|F_{\sigma-1}^{(4)}\right\| \leq d_{\sigma-1} n_{\sigma}^{\epsilon}
$$

and

$$
c_{2} b_{n_{\sigma-1}}>2 n_{\sigma}^{-\epsilon}
$$

Now put

$$
\Phi_{\sigma}(x):=\sum_{j=\sigma}^{\infty} d_{j-1} f_{n_{j}}(x),
$$

where the uniform convergence of the series is justified by (3.2) and the inequality

$$
\left\|\Phi_{\sigma}\right\| \leq \sum_{j=\sigma}^{\infty} d_{j-1} \leq d_{\sigma-1}\left(1+\frac{1}{4}+\frac{1}{4^{2}}+\ldots\right)<2 d_{\sigma-1} .
$$

So we define

$$
f(x):=\sum_{j=1}^{\infty} d_{j-1} f_{n_{j}}(x)=F_{\sigma-1}(x)+\Phi_{\sigma}(x),
$$

and note, that (4.3) and (4.4) yield

$$
f \in \Delta^{(0)}\left(Y_{s}\right) .
$$

Recall also that $g_{b}$ and, hence, $f_{n}$, are odd functions. Therefore, $f$ is odd as well, which implies (4.2).

It remains to verify (4.1). Inequalities (4.5), (4.10), and (4.6) lead to

$$
\omega_{4}\left(\Phi_{\sigma}, 1 / n_{\sigma}\right) \leq c_{1}\left(1+\frac{1}{d^{4}}\right) \frac{1}{n_{\sigma}^{4}} \sum_{j=\sigma}^{\infty} d_{j-1}<2 c_{1}\left(1+\frac{1}{d^{4}}\right) \frac{d_{\sigma-1}}{n_{\sigma}^{4}}<2 \frac{d_{\sigma-1}}{n_{\sigma}^{4-\epsilon}},
$$

as well as (4.8) provides

$$
\omega_{4}\left(F_{\sigma-1}, \frac{1}{n}{ }_{\sigma}\right) \leq \frac{1}{n_{\sigma}^{4}}\left\|F_{\sigma-1}^{(4)}\right\| \leq \frac{d_{\sigma-1}}{n_{\sigma}^{4-\epsilon}}
$$

Hence, for all $\sigma$,

$$
\omega_{4}\left(f, \frac{1}{n}{ }_{\sigma}\right) \leq 3 \frac{d_{\sigma-1}}{n_{\sigma}^{4-\epsilon}}
$$

Finally, let us prove that if $T_{n_{\sigma}} \in \mathbb{T}_{n_{\sigma}} \cap \Delta^{(0)}\left(Y_{s}\right)$, then

$$
\left\|f-T_{n_{\sigma}}\right\| \geq d_{\sigma-1}\left(\frac{b_{n_{\sigma}}^{2}}{n_{\sigma}^{1+\varepsilon}}-3 b_{n_{\sigma}}^{3}\right),
$$


NEGATIVE RESULTS IN COCONVEX APPROXIMATION OF PERIODIC FUNCTION\$

$$
E_{n_{\sigma}}^{(0)}\left(f, Y_{s}\right) \geq d_{\sigma-1}\left(n_{\sigma}^{-\varepsilon-11 / 3}-3 n_{\sigma}^{-4}\right) .
$$

Indeed, since by (3.1) $F_{\sigma-1}$ is zero on $\left[-b_{n_{\sigma-1}}, b_{n_{\sigma-1}}\right]=: J_{\sigma}$, we may write

$$
f(x)=d_{\sigma-1} f_{n_{\sigma}}(x)+\Phi_{\sigma+1}(x), \quad x \in J_{\sigma} .
$$

Let $\tau_{n_{\sigma}}:=T_{n_{\sigma}} / d_{\sigma-1}$. Since $\tau_{n_{\sigma}} \in \Delta^{(0)}\left(Y_{s}\right)$, we have $\tau_{n_{\sigma}}^{\prime}(0) \geq 0$, therefore by virtue of (3.6)

$$
\left\|f_{n_{\sigma}}-\tau_{n_{\sigma}}\right\|_{J_{\sigma}} \geq c_{2} \frac{b_{n_{\sigma}}^{2}}{n_{\sigma}} b_{n_{\sigma-1}}-3 b_{n_{\sigma}}^{3} .
$$

On the other hand, (3.2), (4.10), and (4.7) yield

$$
\left\|\Phi_{\sigma+1}\right\|<2 d_{\sigma}=\frac{c_{2}}{2} \frac{b_{n_{\sigma-1}} b_{n_{\sigma}}^{2}}{n_{\sigma}} d_{\sigma-1} .
$$

Hence

$$
\begin{aligned}
\left\|f-T_{n_{\sigma}}\right\| & \geq\left\|T_{n_{\sigma}}-f\right\|_{I_{\sigma}} \geq\left\|T_{n_{\sigma}}-d_{\sigma-1} f_{n_{\sigma}}\right\|_{I_{\sigma}}-\left\|\Phi_{\sigma+1}\right\| \\
& =d_{\sigma-1}\left\|\tau_{n_{\sigma}}-f_{n_{\sigma}}\right\|_{I_{\sigma}}-\left\|\Phi_{\sigma+1}\right\| \geq d_{\sigma-1}\left(\frac{c_{2} b_{n_{\sigma-1}} b_{n_{\sigma}}^{2}}{2 n_{\sigma}}-3 b_{n_{\sigma}}^{3}\right) .
\end{aligned}
$$

Now (4.14) follows from (4.9). Thus, (4.13) and (4.15) lead to

$$
\frac{E_{n_{\sigma}}^{(0)}(f)}{\omega_{4}\left(f, 1 / n_{\sigma}\right)} \geq \frac{1}{3} n_{\sigma}^{1 / 3-2 \epsilon}-\frac{1}{n_{\sigma}^{\varepsilon}}
$$

for all $\sigma$, that implies (4.1), since the chosen $\varepsilon$ is sufficiently small.

\section{Negative Results in COMOnotone approximation, Proofs of}

\section{THEOREMS 1.2 AND 1.3}

We say that a $2 \pi$-periodic function $f \in C(\mathbb{R})$ is piecewise monotone with respect to $Y_{s} \in \mathbb{Y}_{s}$, if it is a nondecreasing function on $\left[y_{1}, y_{0}\right]$ and it changes its monotonicity at the points $Y_{s}$, that is, if $(-1)^{i-1} f$ is nondecreasing on $\left[y_{i}, y_{i-1}\right], 1 \leq i \leq 2 s$. We denote by $\Delta^{(1)}\left(Y_{s}\right)$ the collection of all such piecewise monotone functions. For a function $g \in \Delta^{(1)}\left(Y_{s}\right)$ denote by

$$
E_{n}^{(1)}\left(g, Y_{s}\right):=\inf _{T_{n} \in \mathbb{T}_{n} \cap \Delta(1)\left(Y_{s}\right)}\left\|g-T_{n}\right\|,
$$

the error of the best comonotone approximation of the function $g$.

First we formulate the well known Lemma 5.1.

Lemma 5.1. For each function $g \in C^{(1)}$ and every polynomial $T_{n} \in \mathbb{T}_{n}$ the inequality

$$
\left\|g^{\prime}-T_{n}^{\prime}\right\| \leq c(k)\left(\omega_{k}\left(g^{\prime}, 1 / n\right)+n\left\|g-T_{n}\right\|\right)
$$

holds, where the constant $c(k)$ depends only on $k$.

Now we prove 
Theorem 5.2. For each $Y_{s} \in \mathbb{Y}_{s}$ there is a function $F \in \Delta^{(1)}\left(Y_{s}\right) \cap C^{(1)}$, such that

$$
\lim _{n \rightarrow \infty} \sup \frac{n E_{n}^{(1)}\left(F, Y_{s}\right)}{\omega_{4}\left(F^{\prime}, 1 / n\right)}=+\infty
$$

Proof. Let $f$ be a function, guaranteed by Theorem 4.1. Identity (4.2) implies, that the function

$$
F(x):=\int_{0}^{x} f(t) d t
$$

is also $2 \pi$ periodic. Then, $F^{\prime} \equiv f$ and $f \in \Delta^{(0)}\left(Y_{s}\right)$ yield $F \in \Delta^{(1)}\left(Y_{s}\right)$. For each polynomial $T_{n} \in \Delta^{(1)}\left(Y_{s}\right) \cap \mathbb{T}_{n}$ (5.1) implies

$$
E_{n}^{(0)}\left(f, Y_{s}\right) \leq\left\|F^{\prime}-T_{n}^{\prime}\right\| \leq c\left(\omega_{4}\left(F^{\prime}, 1 / n\right)+n\left\|F-T_{n}\right\|\right),
$$

whence

$$
n E_{n}^{(1)}\left(F, Y_{s}\right) \geq c E_{n}^{(0)}\left(f, Y_{s}\right)-\omega_{4}(f, 1 / n) .
$$

Therefore

$$
\limsup _{n \rightarrow \infty} \frac{n E_{n}^{(1)}\left(F, Y_{s}\right)}{\omega_{4}\left(F^{\prime}, 1 / n\right)} \geq c \limsup _{n \rightarrow \infty} \frac{E_{n}^{(0)}\left(f, Y_{s}\right)}{\omega_{4}(f, 1 / n)}-1=+\infty .
$$

We are ready to prove Theorem 1.2.

Proof of Theorem 1.2. Let $F$ be a function, guaranteed by Theorem 5.2. Since $F$ is a $2 \pi$-periodic function and $F \in \Delta^{(1)}\left(Y_{s}\right) \cap C^{(1)}$, the function

$$
f(x):=\int_{0}^{x} F(t) d t-\frac{x}{2 \pi} \int_{0}^{2 \pi} F(t) d t
$$

is also $2 \pi$-periodic and $f \in \Delta^{(2)}\left(Y_{s}\right) \cap C^{(2)}$. For each polynomial $T_{n} \in$ $\Delta^{(2)}\left(Y_{s}\right) \cap \mathbb{T}_{n}(5.1)$ implies

$$
\begin{aligned}
E_{n}^{(1)}\left(F, Y_{s}\right) & \leq\left\|f^{\prime}-T_{n}^{\prime}\right\| \leq c\left(\omega_{5}\left(f^{\prime}, 1 / n\right)+n\left\|f-T_{n}\right\|\right) \\
& \leq c\left(\frac{1}{n} \omega_{4}\left(f^{\prime \prime}, 1 / n\right)+n\left\|f-T_{n}\right\|\right),
\end{aligned}
$$

whence

$$
n^{2} E_{n}^{(2)}\left(f, Y_{s}\right) \geq c n E_{n}^{(1)}\left(F, Y_{s}\right)-\omega_{4}\left(f^{\prime \prime}, 1 / n\right) .
$$

Therefore

$$
\limsup _{n \rightarrow \infty} \frac{n^{2} E_{n}^{(2)}\left(f, Y_{s}\right)}{\omega_{4}\left(f^{\prime \prime}, 1 / n\right)} \geq c \limsup _{n \rightarrow \infty} \frac{n E_{n}^{(1)}\left(F, Y_{s}\right)}{\omega_{4}\left(F^{\prime}, 1 / n\right)}-1=+\infty .
$$

Our last formulation is

Theorem $5.3([2])$. For each $Y_{s} \in \mathbb{Y}$ there is a function $f \in \Delta^{(1)}\left(Y_{s}\right)$, such that

$$
\limsup _{n \rightarrow \infty} \frac{E_{n}^{(1)}\left(f, Y_{s}\right)}{\omega_{3}(f, 1 / n)}=+\infty .
$$


Proof of Theorem 1.3. In fact we repeat the previous proof with minor changes. So, Let $F$ be a function, guaranteed by Theorem 5.3. Since $F$ is a $2 \pi$-periodic function and $F \in \Delta^{(1)}\left(Y_{s}\right)$, the function

$$
f(x):=\int_{0}^{x} F(t) d t-\frac{x}{2 \pi} \int_{0}^{2 \pi} F(t) d t
$$

is also $2 \pi$-periodic and $f \in \Delta^{(2)}\left(Y_{s}\right) \cap C^{(1)}$. For each polynomial $T_{n} \in$ $\Delta^{(2)}\left(Y_{s}\right) \cap \mathbb{T}_{n}(5.1)$ implies

$$
E_{n}^{(1)}\left(F, Y_{s}\right) \leq\left\|f^{\prime}-T_{n}^{\prime}\right\| \leq c\left(\omega_{3}\left(f^{\prime}, 1 / n\right)+n\left\|f-T_{n}\right\|\right)
$$

whence

$$
n E_{n}^{(2)}\left(f, Y_{s}\right) \geq c E_{n}^{(1)}\left(F, Y_{s}\right)-\omega_{3}(F, 1 / n)
$$

Therefore

$$
\limsup _{n \rightarrow \infty} \frac{n E_{n}^{(2)}\left(f, Y_{s}\right)}{\omega_{3}\left(f^{\prime}, 1 / n\right)} \geq c \limsup _{n \rightarrow \infty} \frac{E_{n}^{(1)}\left(F, Y_{s}\right)}{\omega_{3}(F, 1 / n)}-1=+\infty,
$$

which is (1.6). Finally, (1.7) follows from (1.6) and the inequality $\omega_{4}(f, t) \leq$ $t \omega_{3}\left(f^{\prime}, t\right)$.

\section{REFERENCES}

[1] H. A. Dzyubenko, Comonotone approximation of twice differentiable periodic functions, Ukr. Math. J. 61 (2009), 519-540. Translated from Ukrainskyi Matematychnyi Zhurnal, Vol. 61, No. 4 (2009), 435-451.

[2] G. A. Dzyubenko, Contrexample in comonotone approximation of periodic functions, Transactions of Institute of Mathematics, the NAS of Ukraine, 5 (2008), No. 1, 113123 (in Ukrainian).

[3] G. A. Dzyubenko, J. Gilewicz and I. A. Shevchuk Piecewise monotone pointwise approximation, Constr. approx. 14 (1998), 311-348.

[4] K. A. Kopotun, D. Leviatan, A. Prymak and I. A. Shevchuk, Uniform and Pointwise Shape Preserving Approximation by Algebraic Polynomials, Surveys in Approximation Theory 5 (2011), 1-51.

[5] D. Leviatan, O.V. Motorna and I. A. Shevchuk, No Jackson-type estimates for piecewise q-monotone, $q \geq 3$, trigonometric approximation, arXiv:2004.03724v1 [math.CA] 7 Apr 2020.

[6] D. Leviatan, I.A. Shevchuk, Coconvex polynomial approximation, Journal of Approximation Theory 121 (2003), 100-118.

[7] G. G. Lorentz and K. L. Zeller, Degree of Approximation by Monotone Polynomials I, J. Approx. Theory, 1 (1968), 501-504.

[8] M. G. Pleshakov, Comonotone Jacksons Inequality, J. Approx. Theory, 99 (1999), 409-421.

[9] A. A. Privalov Theory of interpolation of functions, Book 1, Saratov University Publishing House, Saratov 1990, (In Russian).

[10] V. D. Zalizko, Coconvex approximation of periodic functions, Ukr. Math. J. 59, (2007), 28-44. Translated from Ukrainskyi Matematychnyi Zhurnal, Vol. 59, No. 1, pp. 29-43, January, 2007. 
(G. Dzyubenko) Institute of Mathematics NAS of Ukraine, 01024 Kyiv, Ukraine

Email address: dzyuben@gmail.com

(V. Voloshyna) Faculty of Mechanics and Mathematics, Taras Shevchenko National University of Kyiv, 01601 Kyiv, Ukraine; University of Toulon, 83130, LA Garde, France

Email address: victoria.voloshyna@yahoo.com

(L. Yushchenko) University of Toulon, 83130, La Garde, France

Email address: lyudmyla.yushchenko@univ-tln.fr 